\title{
CLAREAMIENTO DENTAL EN DIENTES VITALES
}

\section{DENTAL CLAREMENT IN VITAL TEETH}

\author{
Pinos-Samaniego Mariana Gabriela. ${ }^{1 *}$ Cevallos-Romero Sandra. ${ }^{2}$ \\ ${ }^{1}$ Odontóloga-Especialista en Rehabilitación Oral.Cuenca.Ecuador. \\ ${ }^{2}$ Odontóloga Rural del Ministerio de Salud Pública del Ecuador.Ecuador. \\ * gabipinos15@hotmail.com
}

\begin{abstract}
Resumen
El objetivo de este estudio fue realizar una revisión de literatura para recopilar información sobre el clareamiento dental en piezas vitales, con el propósito de ofrecer al profesional información relevante sobre el tipo de clareamiento dental, contraindicaciones, limitaciones, principios básicos del clareamiento, complicaciones clínicas, efectos adversos y toxicidad, ya que en la actualidad este tipo de tratamiento es el más frecuente en las consultas de odontólogos. Se realizó una búsqueda bibliográfica en PubMed con las palabras clave "blanqueamiento dientes vitales", en artículos publicados desde el 2003 hasta 2016. Las fuentes consultadas para esta revisión fueron 394 artículos, de los cuales 28 fueron incluidos en la revisión luego de aplicar criterios de exclusión como: estudios realizados en humanos, ensayos clínicos aleatorizados, revisiones de la literatura y revisiones sistemáticas, a más de 1 libro y 3 boletines asociado al blanqueamiento dental. Se observó que existe una alta demanda de pacientes deseosos por obtener una sonrisa blanca y brillante, la sonrisa se ha convertido en el primer contacto para la comunicación entre las personas, una bella sonrisa establece la mejor carta presentación, por lo cual la estética dental ha sido una inquietud constante en las diversas civilizaciones hasta la actualidad. Es un procedimiento que, utiliza sustancias químicas como el peróxido de sodio y el peróxido de carbamida, reduce la presencia de manchas en piezas dentales vitales y no vitales, removiendo por ende los pigmentos que se encuentran depositados en las mismas.
\end{abstract}

Palabras clave: Peróxido de carbamida, insicivo, peróxido de hidrógeno.

\begin{abstract}
The objective of this study was to conduct a literature review to collect information on dental cleansing in vital parts, with the purpose of offering the professional relevant information on the type of dental clearing, contraindications, limitations, basic principles of cleansing, clinical complications, adverse effects and toxicity, since at present this type of treatment is the most frequent in the dental offices. A bibliographic search was conducted in PubMed with the keywords "whitening vital teeth", in articles published from 2003 to 2016. The sources consulted for this review were 394 articles, of which 28 were included in the review after applying criteria of exclusion as: human studies, randomized clinical trials, reviews of literature and systematic reviews, more than 1 book and 3 bulletin associated with teeth whitening. It was observed that there is a high demand for patients anxious to obtain a bright white smile, since the smile is the first contact for the approach and communication between people, a beautiful smile establishes the best presentation letter, so the aesthetics Dental has been a constant concern during history in various civilizations to this day. It is a procedure that uses chemical substances such as sodium peroxide and carbamide peroxide, reduces the presence of stains on vital and non-vital teeth, thus removing the pigments that are deposited therein.
\end{abstract}

Key words: Carbamide peroxide, incisive, hydrogen peroxide.

\section{INTRODUCCIÓN}

Según la OMS, "la salud es un estado de completo bienestar físico, mental y social y no solamente la ausencia de afecciones o enfermedades". ${ }^{2}$ Por lo que es indudable que en el caso de pacientes que presentan dientes oscuros especialmente en el sector anterior, es normal que estos se sientan afectados, ya que durante la comunicación entre las personas tener una bella sonrisa genera seguridad en las personas, una sonrisa estética establece la mejor carta presentación. El acto de sonreír, se ve restringido por un mal color dental, esto provoca no solo un problema estético sino también de bienestar. Por lo tanto, hoy en día las consultas de odontólogos tienen mayor frecuencia de pacientes deseosos por obtener una sonrisa blanca y brillante. ${ }^{2}$

Desde las antiguas civilizaciones hasta nuestros días la belleza ha preexistido como un anhelo permanente por el ser humano. Existen evidencias de que los Mayas de América Central y del Sur (en torno al año 1000 d.C.) decoraban la 
boca alisando los bordes incisales de piezas dentales anteriores con numerosas formas y diseños. ${ }^{1}$

La limpieza bucal tuvo una gran significancia durante el Imperio Romano, el tratamiento estético era un servicio en el que únicamente las clases pudientes podían acceder. A pesar de esto era habitual el uso de enjuagues bucales, dentífricos y palillos de dientes. La estética dental ha sido un anhelo permanente desde la antigüedad hasta nuestros días. ${ }^{1}$

El filósofo Umberto Eco en su obra "Historia de la Belleza", nos relata como el hombre ha ido cambiando la percepción de belleza a través del tiempo y lo que antes era considerado bello se ve amenazado en la actualidad por la publicidad por lo que el concepto de belleza en el mundo de hoy es un concepto muy relativo. El hombre se ha transformado en una mera copia de lo que establecen los medios. Con esto podemos hacernos la pregunta de sí la belleza es lo que nos genera algo cuando lo observamos en un primer instante, o sí en verdad es bello solo lo que la sociedad considera como tal. $^{3}$

Teniendo como referencia estos conocimientos históricos es entendible que los métodos y productos de blanqueamiento sigan en estudio y desarrollo hasta nuestra actualidad. Antes de abordar en las técnicas de clareamiento dental, es importante conocer porque se producen las decoloraciones en las piezas dentales, las mismas que aparecen cuando hay anomalías en la calcificación de las mismas, presentando alteraciones en sus estructuras y mineralización dental. ${ }^{1}$

El clareamiento dental es el proceso en que se aplica un gel clareador sobre la superficie dentaria por un tiempo, es una solución estética para los pacientes que presentan pigmentaciones en la superficie del diente, el color natural del diente es influenciado por la trasmisión de luz y las propiedades de reflexión del tejido duro del diente, el color del diente está determinado por las propiedades que presenta la dentina. Este color dental puede ser modificado por colorantes denominados cromóforos, que pueden ser extrínsecos o intrínsecos. ${ }^{4,5}$

La etiología puede ser de tipo endógenas (hereditarias), las mismas que afectan el esmalte y la dentina tanto en la dentición primaria como a la secundaria y tienen una orientación difusa o incluso vertical. Las exógenas(ambientales), en cambio, afectan a uno o a un grupo de dientes, en su esmalte como en la dentina estas se presentan con una orientación horizontal. ${ }^{4,5}$

Hay diferentes tipos de manchas, las cuales aparecen por diversas causas, por ejemplo: los componentes salivales pueden ocasionar manchas pardas, la clorofila puede provocar manchas verdes, una dentinogenesis imperfecta puede originar manchas pardo violeta, amarillentas o gris. Existen también manchas de etiología desconocida. ${ }^{4,5}$

El tipo de clareamiento dental dependerá de la etiología del cromóforo, pudiendo ser una limpieza profesional suficiente para la eliminación de la mayoría de machas externas, el tratamiento para cromóforos internos es más compleja y se basa en diferentes métodos como la macroabrasión, usada para restaurar a través de cerámicas o composites, técnicas de microabrasión (ácido clorhídrico al 18\%) y el blanqueamiento dental. ${ }^{4,6,8}$

El clareamiento dental puede ser intracoronal en dientes sometidos a endodoncia (blanqueamiento en dientes no vitales) o externo (clareamiento en dientes vitales). A pesar de un largo número de métodos descritos en la literatura sobre el blanqueamiento externo de dientes vitales, la mayoría de estos se basan con el uso directo del peróxido de hidrogeno $\mathrm{H} 2 \mathrm{O} 2$, o su precursor el peróxido de carbamida. ${ }^{9}, 11$

Este estudio ha realizado una búsqueda literaria exhaustiva, con el objetivo de analizar los conceptos fundamentales sobre claremiento dental en piezas vitales. Se realizó una búsqueda bibliográfica en PubMed con las palabras clave "blanqueamiento dientes vitales", en artículos publicados desde el 2003 hasta 2016 encontrando 389 artículos, de los cuales 23 fueron incluidos en la revisión luego de aplicar criterios de exclusión como: estudios realizados en humanos, ensayos clínicos aleatorizados, revisiones de la literatura y revisiones sistemáticas, a más de 1 libro y 1 boletín asociado al blanqueamiento dental.

\section{Estado del Arte}

\subsection{Principios básicos del clareamiento.}

El clareamiento disuelve las largas cadenas moleculares de las manchas entre el esmalte y la dentina, que con el paso del tiempo forman moléculas más grandes y hacen que el diente aparezca más obscuro. La capa semipermeable que existe en la superficie del esmalte y dentina permite la absorción de líquido que contiene moléculas muy pequeñas de los cromóforos, capaces de pasar a través de la membrana semipermeable y penetrar en el esmalte y dentina. ${ }^{4,9,13}$

Los colorantes entran y salen a través de la membrana, las moléculas largas no entran por ser muy grandes y no pasan a través de los poros, en el interior de los túbulos dentinarios y la dentina intertubular, las moléculas pequeñas del cromóforo tienden a unirse entre sí formando enlaces dobles, convirtiéndose en una cadena molecular larga, imposible de salir a través de los poros de la membrana semi permeable, quedándose atrapada en el diente. Con el pasar del tiempo estas manchas crecen tanto en esmalte y dentina, haciendo que la estructura del diente aparezca progresivamente más obscura. ${ }^{4,9,13}$

Los cromóforos pueden ser tratados con una variedad de materiales para el clareamiento, el más común incluye peróxido de hidrogeno y peróxido de carbamida. El peróxido de carbamida es una forma mejorada del peróxido de hidrogeno, menos caustico para el tejido blando, libera significativamente menos iones oxigeno clareadores en la superficie del diente este se aplica a la superficie y rompe rápidamente en carbamida y peróxido. La carbamida es un producto común de la dieta, que podemos encontrarlo rutinariamente en el sistema 
digestivo, esta pasa a través del sistema humano sin producir ningún efecto, con la excepción de producir flatulencia. En cambio, el peróxido se descompone en molécula de agua e ion oxígeno que son fácilmente procesada por el sistema digestivo. $4,9,13$

Entonces la carbamida y el agua se eliminan sin producir daño, dejando los iones oxígeno en las proximidades de la superficie del diente, cuando los iones de oxigeno penetran a través de la superficie del diente lo hacen entre 5 y 15 minutos y destruyen el doble enlace que se creó entre las moléculas del cromóforo impidiéndola salir a través de la membrana semipermeable, reduciendo al colorante en su pequeña constitución original, permitiéndolo pasar por la a la superficie, lo que permite al colorante salir del esmaltedentina. En un corto tiempo, la mayor parte del cromóforo ha salido de la estructura dentaria, este se lava y enjuaga al diente con agua. Se le debe indicar al paciente que debe cepillarse y usar el hilo dental para remover las moléculas de colorante que pudieran haber quedado y para mantener su clareamiento. ${ }^{4,9,13}$

\subsection{Indicaciones}

El clareamiento dental consigue dar excelentes resultados, aplicándolos en las condiciones adecuadas y dentro de las indicaciones permitidas, la única indicación necesaria es el requerimiento de clareamiento dental por parte del paciente (Necesidad sentida). ${ }^{31}$ Las técnicas de clareamiento son utilizadas para tratar algunos de los siguientes casos (Tabla 1)..$^{9,12,13}$

\subsection{Contra-indicaciones}

Hay pocas contraindicaciones para el clareamiento dental, tenemos:

- Pacientes con expectativas muy altas. ${ }^{13}$

- Pacientes alérgicos a alguno de los componentes del producto (que prácticamente son inexistentes).

- Las mujeres embarazadas deberían evitar cualquier procedimiento estético que puede dañar el feto o recién nacido, por lo que más vale prevenir que lamentar.

- Para la decisión de usar un tratamiento casero o en la clínica, se debe tener en cuenta la amplitud de la cámara pulpar o si el paciente ha presentado problemas de sensibilidad previos, los cuales deberían ser resueltos antes de comenzar cualquier tratamiento.

- Pacientes con erosiones, abrasiones, recesiones pueden experimentar mayor sensibilidad durante o después del tratamiento, por lo que esas erosiones deberían ser tratadas antes, lo mismo debería hacerse con los que presentan abfraciones. ${ }^{9}$

- Existen coronas o restauraciones que necesitan ser cambiadas después del blanqueamiento, debemos considerar esto como una contraindicación, puesto que existe pacientes que no quieren hacerlo o no pueden cubrir el costo de estas.
Tabla 1.: Indicaciones para un realizar un clareamiento dental.

1.Manchas adquiridas por consumo de café de tè o por fumar.

2.Manchas en el esmalte y en la dentina.

3.Fluorosis.

4. Manchas por Tetraciclinas.

5. Dientes amarillentos por la edad.

6. Tratamientos pre y post restaurativos.

7. Cambios producidos por una pulpa traumatizada.

- Pacientes de edad avanzada con exposición de las raíces amarillas representan un problema ya que las raíces no se aclaran con la misma facilidad en comparación con las coronas, dejando un desajuste evidente que requiere ser corregida por una restauración. ${ }^{13}$

\subsection{Limitaciones}

Los factores que pueden limitar el éxito del clareamiento son el grado y calidad de la decoloración. Si son los dientes extremadamente obscuros, no importa cuál sea la causa, el clareamiento podría requerir complementarse con una restauración como una carilla de porcelana. Es verdad también que las manchas en la escala entre gris y azul, no responden muy bien como aquellas manchas entre amarillas y cafés. ${ }^{9}$ Pacientes con una expectativa muy alta nunca estarán satisfechos, estos pacientes pueden ser claramente identificados con una simple pregunta: ¿Qué es lo que esperaran alcanzar con el blanqueamiento dental? Y aquellos que respondan que pretenden conseguir un blanco deslumbrante pertenecen a este grupo de pacientes, ${ }^{12}$ tratar de conseguir un óptimo resultado para el paciente puede requerir aplicaciones más largas del tratamiento o múltiples aplicaciones, con las cuales lo único que conseguiremos es incrementar el riesgo de sensibilidad dental. A estos pacientes se les puede explicar que todo diente tiene un límite de cuán rápido puede cambiar el color, y cuan brillante puede llegar a ser. ${ }^{15}$

\subsection{Complicaciones clínicas y efectos adversos}

Peróxido de hidrógeno es un derivado de oxígeno térmicamente inestable, cuyos radicales libres derivados de la degradación como son el ion hidroxil $\left(\mathrm{OH}^{*}\right)$, en contacto con las células, inicia una cadena de oxidación de los fosfolípidos presentes en los lisosomas y membrana del citoplasma (peroxidación lípida), con una consecuente salida de enzimas y daño a las células pulpares causando muerte celular, con lo que se explica la necrosis que ocurre en el tejido pulpar después del clareamiento dental. El principio activo es su acción como agente oxidativo capaz de degradar moléculas orgánicas complejas, que son las responsables del cambio de coloración del diente. Por su bajo peso molecular del H2O2 lo hace capaz de difundirse a través de esmalte y dentina para llegar al espacio pulpar. El $70 \%$ de los pacientes sometidos 
a clareamiento se quejan de la sensibilidad postoperatoria, especialmente en dientes anteriores, esto ocurre debido a la variación del grosor del esmalte y la dentina. ${ }^{16,17}$

\subsection{Acción del peróxido de hidrógeno sobre la estructura de esmalte y dentina}

Los radicales libres, afectan a las moléculas pigmentadas, pero también van afectar a los componentes orgánicos de los tejidos duros del diente. En la superficie dental se ha podido observar rugosidades en la superficie, así como el aumento discreto e irregular de porosidades en la misma. ${ }^{4,17}$ Los productos utilizados para el blanqueamiento dental provocan efectos adversos a la estructura del esmalte dental, los mismos que dependerán de la metodología empleada como: tipo de dientes, medio de almacenamiento, tiempo de exposición, composición de los agentes de blanqueamiento comercializados, y $\mathrm{pH}$ de la solución. ${ }^{1}$ En los diferentes geles de blanqueamiento dental disponibles en el mercado sea podido observar que existe una gran variación en el $\mathrm{pH}$ de los mismos, por lo que se asumido que tanto la disminución de la microdureza del esmalte como la alteración de la morfología de la superficie pueden atribuirse, a las propiedades acidógenas de los agentes de blanqueamiento. Sin embargo, los defectos observados después del blanqueamiento de dientes vitales son:

1. Menos severos que los producidos por la aplicación de gel de ácido fosfórico al $37 \% .^{21}$

2. No parecen aumentar la susceptibilidad del esmalte a la caries. ${ }^{22}$

Existen varios estudios donde nos indican que los efectos producidos por los productos blanqueadores pueden ser neutralizados por el potencial de remineralización de la saliva. (16) La utilización de flúor el cual se encuentra en diversos productos de blanqueamiento estos nos ayudaran a que se dé un efecto de remineralización provocando que se dé un aumento de la microdureza del esmalte durante el tratamiento y el período de postratamiento. ${ }^{4}$

\subsection{Acción de los productos de blanqueamiento dental so- bre la adhesión al esmalte y la dentina.}

Al examinar la adhesión de los composites al esmalte en las piezas dentales que se han realizado un blanqueamiento, se ha observado que existe una disminución en la resistencia de la unión adhesiva esmalte-resina cuando el procedimiento de adhesión se ejecuta seguidamente después del blanqueamiento de dientes vitales esto es independiente de la concentración de peróxido de hidrógeno o de carbamida empleada. $^{23}$

Los productos de blanqueamiento dental se usan habitualmente sobre la estructura del esmalte la misma que al ser permeable va afectar a la dentina, esto ocurre tanto en el blanqueamiento realizado en un consultorio, como en el blanqueamiento ambulatorio, la alteración en la adhesión que estos productos causan puede desaparecer con el pasar del tiempo. Diversos estudios nos muestran que los valores de resistencia adhesiva dental regresan a niveles normales entre 24 h y 3 semanas después del ver realizado el blanqueamiento. ${ }^{4}$

\subsection{Acción del peróxido de hidrógeno sobre la pul- pa:Sensibilidad dentaria.}

Podríamos decir que la sensibilidad dentaria es el principal efecto adverso del blanqueamiento de dientes vitales, lo cual ha sido evidenciado en diferentes estudios, así tenemos que según el estudio de Haywood et al, un $52 \%$ de los pacientes que se sometieron a blanqueamiento dental en dientes vitales con férula nocturna y peróxido de carbamida al $10 \%$ durante 6 semanas experimentaron sensibilidad dentaria. Existen varios estudios donde señalan que la sensibilidad dentaria es transitoria y desaparece al finalizar el tratamiento o poco después. ${ }^{23}$ El mayor índice de sensibilidad se mostrará al principio del tratamiento de blanqueamiento, por tal motivo se ha planteado la utilización de agentes desensibilizantes, como el nitrato potásico y el flúor para reducir la sensibilidad dentaria la misma que se genera por la penetración del agente blanqueador en la cámara pulpar dando lugar a una pulpitis reversible. ${ }^{4}$

El peróxido de hidrógeno, utilizado de forma directa o derivado de la aplicación de peróxido de carbamida, se propaga a través del esmalte a la dentina y de esta hacia el interior de la cámara pulpar, incluso con tiempos de exposición cortos de $15 \mathrm{~min}$. Esto lo ha podido demostrar los diferentes experimentos in vitro realizados. Además, Gokay et al encontraron que la cantidad de penetración del peróxido de hidrógeno en el interior de la cámara pulpar de los dientes restaurados era más elevada que en dientes sanos y que se veía influida por el tipo de material restaurador. ${ }^{4}$

Después de un blanqueamiento vital con férula nocturna con peróxido de carbamida al $10 \%$ durante dos semanas, Fugaro et al describieron una reacción pulpar leve y localizada, sin aumento de los marcadores inflamatorios. Los leves cambios histológicos observados revirtieron en las siguientes dos semanas después del tratamiento. ${ }^{4}$

Seale et al, sin embargo, advirtieron en su estudio in vivo sobre perros que la aplicación de peróxido de hidrógeno al $35 \%$ durante $30 \mathrm{~min}$, en cada uno de los 4 períodos semanales, causaba en los tres días siguientes después del tratamiento una respuesta pulpar muy marcada en el área inmediatamente por debajo de la superficie de esmalte tratada. Sesenta días después de terminado el tratamiento se observaba una resolución de esta respuesta inflamatoria. En este estudio, los daños causados por el peróxido de hidrógeno no se vieron intensificados por la adición de calor de $62{ }^{\circ} \mathrm{C}$; además, no se observaron efectos nocivos sobre la pulpa tras la aplicación sólo de calor. ${ }^{4}$

Eldeniz et al y Sulleman et al manifestaron en su investigación in vitro que en el $15 \%$ de los dientes de los monos 
Rhesus, se producía un daño pulpar irreversible al utilizar una lámpara láser de diodo la misma que provoca un aumento de la temperatura intrapulpar que superaba el umbral crítico de $5,6^{\circ} \mathrm{C} .^{4}$

Por otro lado, la pulpa tiene la capacidad de protegerse así misma frente a los daños causados por el peróxido de hidrógeno. Así, Lee et al han demostrado que el tratamiento con peróxido de hidrógeno a concentraciones por debajo de 0,3 $\mathrm{mmol} / \mathrm{l}$ aumenta la capacidad de los odontoblastos de producir dentina. Por tanto, parece que los odontoblastos pueden reaccionar en cierta medida a la acción del peróxido de hidrógeno y que los mecanismos de la pulpa protegen a este tejido de los radicales generados por el peróxido de hidrógeno. Estos mecanismos pueden contribuir a la reversibilidad tanto de los daños fisiológicos como de la sensibilidad dentinaria. $^{4}$

\subsection{Carcinogenicidad de los productos de blanqueamiento}

A la luz de todos los estudios animales que se han llevado a cabo, la Agencia Internacional de Investigación sobre el Cáncer (AIIC) ha hablado sobre el potencial carcinogénico del peróxido de hidrógeno y ha concluido que la evidencia es limitada en el caso de los animales de experimentación e inadecuada en humanos. La AIIC basa sus conclusiones en el hecho de que el débil efecto de estimulación, promoción y/o carcinogénesis demostrado en algunos estudios animales ocurrió tras la exposición repetida a elevadas dosis de peróxido de hidrógeno, lo que hace que estos estudios no sean representativos de las bajas cantidades de peróxido de hidrógeno que se emplean en el blanqueamiento dental. Por lo que se requiere ahora un estudio clínico a gran escala para excluir de forma definitiva el riesgo carcinogenético en pacientes. $^{4}$

\subsection{Efecto adverso de la luz, calor y láser en el clarea- miento dental}

La literatura nos refiere que el uso de calor, luz o laser no está justificado para conseguir mayor clareamiento en la pieza dental, sino que más bien lleva al incremento de la temperatura intrapulpar con un valor crítico del $5,5^{\circ} \mathrm{C}$, con el que conseguimos el daño al tejido pulpar, siempre y cuando el iniciador del producto no sean las canforoquinomas, caso contrario su uso estaría contraindicado. ${ }^{16,26}$

\subsection{Toxicidad}

Se ha reseñado en los estudios clínicos la irritación gingival como efecto adverso común del blanqueamiento vital con férula nocturna, por lo que es aconsejable que la férula cubra únicamente al esmalte. Y cuando el blanqueamiento vital se realiza en la consulta con peróxido de hidrógeno a elevada concentración, se recomienda proteger de forma mecánica la mucosa gingival mediante dique de goma con ligaduras y crema aislante. ${ }^{25}$
El efecto agudo de los blanqueantes dentales comercializados depende de la cantidad y la concentración ingeridas. Sin embargo, el uso de los productos de blanqueamiento dental que contienen peróxido de carbamida al $35 \%$ está restringido a la técnica de aplicación en la consulta dental y por lo tanto se emplean siempre bajo supervisión clínica. ${ }^{25}$

La toxicidad de los agentes de blanqueamiento dental por lo tanto guarda más relación con su ingestión accidental, como en el caso por ejemplo de accidentes con niños pequeños, por lo que es importante mantener estos productos fuera del alcance de los niños para evitar accidentes, a pesar de que no se ha descrito ningún caso en la literatura sobre envenenamiento con clareadores dentales. ${ }^{25}$

\section{DISCUSIÓN}

El presente estudio se realizó mediante una revisión de literatura para recopilar información sobre el clareamiento dental en piezas vitales, con el propósito de ofrecer al profesional información relevante sobre el tema, este tipo de tratamiento en la actualidad es el más frecuente en las consultas de dentistas y odontólogos. Se consultaron 394 artículos, de los cuales 28 fueron incluidos en la revisión luego de aplicar criterios de exclusión como: estudios realizados en humanos, ensayos clínicos aleatorizados, revisiones de la literatura y revisiones sistemáticas, a más de 1 libro y 3 boletines asociado al blanqueamiento dental. Se observó que existe una alta demanda de pacientes ansiosos por obtener una sonrisa blanca y brillante.

Caballero, A, Forner L, Amengual J. en su estudio Blanqueamiento vital domiciliario: comparación de tratamientos con peróxido de hidrógeno y peróxido de carbamida, realizado a 6 personas de las cuales 3 fueron tratadas con uno de los productos mencionados y los otros 3 con el otro, demostró que ambos productos son eficaces en su función blanqueadora para lo que han sido desarrollados y la hipersensibilidad dentaria es mínima. ${ }^{27} \mathrm{Al}$ compararlo con el estudio realizado por Eustáquio J, Ramos A. se observó que la técnica de blanqueamiento asociada con el uso del gel blanqueador a base de peróxido de hidrogeno de $35 \%$ (Clàriant Angelus Office $35 \%$, Angelus) en el consultorio, y el gel blanqueador a base de peróxido de carbamida de $16 \%$ casero (Clàriant Angelus Home 16\%, Angelus) produjo, en ese caso, resultados satisfactorios en blanquear los dientes naturales, vitales y amarillados, sin causar la sensibilidad dentaria. Por lo que existe una similitud en los resultados de ambos estudios. ${ }^{28}$

Al contrario que los anteriores, estudios realizados Ortíz M, Zavala N, Patiño N, Martínez G, Ramírez J. en su investigación confirman que el peróxido de carbamida al $15 \%$ utilizado como blanqueador sí afecta la micromorfología de la superficie del esmalte, causando porosidades, además de la disminución de su microdureza. ${ }^{29}$ Asi mismo Lozada O, García C, concluyeron en su estudio que el blanqueamiento dental es un tratamiento que a pesar de ser conservador 
y generalmente dar resultados satisfactorios, presenta una serie de riesgos dentro de los cuales los más comunes son: la sensibilidad dentaria postoperatoria, y la longevidad de los resultados. ${ }^{30}$ Los profesionales deben considerar estos factores antes de realizar un blanqueamiento dental, para informar y prevenir a los pacientes de los síntomas que se pueden presentar, ya que en la actualidad este tratamiento se ha convertido en una necesidad para la mayoría de la población.

\section{CONCLUSIÓN}

El clareamiento dental es un procedimiento estético, cuya evidencia apoya la seguridad de uso del peróxido de hidrógeno en dientes vitales cuando el procedimiento es su-12 pervisado clínicamente. Es importante diferenciar la etiología del cromóforo para elegir el procedimiento más indicado, seguros de que los productos no inducen alteraciones im-13 portantes sobre las estructuras de esmalte y dentina o en caso de producirse alguna son muy pequeñas y pueden ser contrarrestadas con la saliva o flúor. En caso de requerir ${ }^{14}$ procedimientos restauradores, estos deben retrasarse al menos $24 \mathrm{~h}$ después del blanqueamiento dental, debido a la pérdida de la adhesión, y la sensibilidad dentaria es uno de los efectos adversos más importantes del blanqueamiento de dientes vitales y refleja una pulpitis reversible ya que ningún estudio ha reseñado efectos de pulpitis irreversible. 15 No existen evidencias a partir de los datos experimentales que el peróxido de hidrógeno empleado en los procedimientos de blanqueamiento en estas dosis presente riesgo de toxicidad y/o carcinogenésis.

\section{Referencias}

1 Huayllucu E. Blanqueamiento de dientes vitales. Rev. Act. ${ }^{17}$ Clin. Med. 2012; 22: 1130-1134.

2 Peydro M. Blanqueamiento dental en dientes vitales $\mathrm{y}$ no vitales. [Internet Blog]. [Consultado 1, jul, 20181]. Disponible en: http://www.sonrisaespectacular.com/2015/07/blanqueamientodental-en-dientes-vitales-y-no-vitales/

3 Eco U. Historia de la Belleza. Editorial Lumen. 1 Edición.

4 Minoux M, Serfaty R. Vital tooth bleaching: Biologic adverse effects-A review. Quintessence Int. 2008;39(8):645-59.

5 Dudea D, Florea A, Mihu C, Campeanu R, Nicola C, Bemga $\mathrm{GH}$. The use of scanning electron microscopy in evaluating the effect of a bleaching agent on the enamel surface. Am J Dent. 2009;50(3):435-40.

6 Noriega A, Salgado M. Tratamiento estético conservador 2 con microabrasión sobre hipoplasias del esmalte en dientes permanentes jóvenes. Rev Tamé. 2014;3(8):271-4.

7 Lynch C, McConnell R. The use of microabrasion to remove discolored enamel. A clinical report. J Prosthet Dent. 2003;90(5):417-9.
8 Price R, Loney R, Doyle M, Moulding M. An evaluation of a technique to remove stains from teeth using microabrasion. 2003;134(8):1066-71.

9 Freedman G. Contemporary Esthetic Dentistry. 1a ed. Elsevier; 2012.341 - 404 p.

10 Moraes RR, Marimon JLM, Schneider LFJ, Correr Sobrinho L, Camacho GB, Bueno M. Carbamide peroxide bleaching agents: effects on surface roughness of enamel, composite and porcelain. Clin Oral Investig. 2006;10(1):23-8.

11 Azrak B, Callaway A, Kurth P, Willershausen B. Influence of Bleaching Agents on Surface Roughness of Sound or Eroded Dental Enamel Specimensjerd 3 72.JEsthetRestorDent,2010;22(6) : $391 \smile 401$.

Alay M, Bosch M. Trastornos dentales inducidos por fármacos. Butlletí de Farmacovigilància de Catalunya. 2009;7(1):1-4.

3 Sulieman M. An overview of tooth-bleaching techniques: chemistry, safety and efficacy. Periodontology 2000. 2008;48:148-69.

4 Parra J, Astudillo D, Cedillo N, Ordoñez G, Sempértegui F. Fluorosis dental: Prevalencia, grados de severidad y factores de riesgo en niños de 7 a 13 años del Cantón Cuenca. Maskana [Internet]. 2015 [citado 31 de enero de 2016];3(1). Recuperado a partir de: http://www.ucuenca.ec/ojs/index.php/maskana/article/view/393 5 He L, Shao M, Tan K, Xu X, Li J. The effects of light on bleaching and tooth sensitivity during in-office vital bleaching: A systematic review and meta-analysis. Journal of dentistry. 2012;40:6 4 4-6 53.

16 Kina J, Huck C, Riehl H, Martinez T, Sacono N, Ribeiro A, et al. Response of human pulps after professionally applied vital tooth bleaching. International Endodontic Journal. 2010;43:572-80,

17 Souza C, Riehl H, Kina J, Tomoko N, Hebling J. Human pulp responses to in-office tooth bleaching. Oral Surg Oral Med Oral Pathol Oral Radiol Endod. 2010;109:e59-64.

8 Lopes GC, Bonissoni L, Baratieri LN, Vieira LC, Monteiro S. Effect of bleaching agents on the hardness and morphology of enamel. J Esthet Restor Dent. 2002;14:24-30.

19 White DJ, Kozak KM, Zoladz JR, Duschner H, Gotz H. Peroxide interactions with hard tissues: Effects on surface hardness and surface/ subsurface ultrastructural properties. Compend Contin Educ Dent. 2002;23:42-8.

20 Yurdukoru B, Akoren AC, Unsal MK. Alterations in human enamel surface morphology following the use of an office bleaching agent and consecutive application of $37 \%$ phosphoric acid in vivo. 2003;14:103-7.

21 Ernst CP, Marroquin BB, Willershausen-Zonnchen B. Effects of hydrogen peroxide-containing bleaching agents on the morphology of human enamel. Quintessence Int. 1996;27:53-6.

22 Al-Qunaian TA. The effect of whitening agents on caries susceptibility of human enamel. Oper Dent. 2005;30:265-70. 
23 Kum KY, Lim KR, Lee CY. Effects of removing residual peroxide and other oxygen radicals on the shear bond strength and failure modes at resintooth interface after tooth bleaching. 2004;17:267-70.

24 Haywood VB, Leonard RH, Nelson CF, Brunson WD. Effectiveness, side effects and long-term status of nightguard vital bleaching. J Am Dent Assoc. 1994;125:1219-26.

25 Giberson T, Kern J. Near-Fatal Hydrogen Peroxide Ingestion. Annals of Emergency Medicine. 1989;18(7):778-9.

26 Buchalla W, Attin T. External bleaching therapy with activation by heat, light or laser. A sistematic review. Dental Materials. 2007;23:586-96

27 Berga A, Forner L, Amengual J .Blanqueamiento vital domiciliario: comparación de tratamientos con peróxido de hidrógeno y peróxido de carbamida. Med. oral patol. oral cir.bucal. 2006; 11( 1 ): 94-99.

28 Eustáquio J, Thereza A. Blanqueamiento dental - Asociación de técnicas para obtener la efectividad y naturalidad. Ángelus*. 55 (43) 2101-3200.

29 Ortíz M,* Zavala N, Patiño N, Martínez G, Ramírez J. Efecto del blanqueamiento y el remineralizante sobre la microdureza y micromorfología del esmalte dental. Revista ADM. 2016; 73 (2): 81-87

30 Lozada O, García C. Riesgos y beneficios del blanqueamiento dental. Acta Odontologica Venezolana. 2000; 38(1).

31 Nuestra necesidad sentida. [en línea]. Bolivia: Opinión; 2014. [Fecha de acceso 3 de julio de 2018].URL disponible en: http://www.opinion.com.bo/opinion/articulos/2014/0625/ noticias.php?id=131405

Recibido: 13 de Junio de 2018.

Aceptado: 17 de Julio 2018. 
